$\begin{array}{cl}\text { PRAMANA } & \text { (C) Indian Academy of Sciences } \\ \begin{array}{c}\text { journal of } \\ \text { physics }\end{array} & \begin{array}{l}\text { Vol. } 62, \text { No. } 3 \\ \text { March 2004 } \\ \text { pp. } 541-544\end{array}\end{array}$

\title{
Search for supersymmetry at LEP-2
}

\section{EHUD DUCHOVNI}

Weizmann Institute of Science, Rehovot 76100, Israel

\begin{abstract}
An extensive effort to search for a hint of a supersymmetric signal was carried out by the four LEP Collaborations. All LEP data, up to centre-of-mass energy of 209 $\mathrm{GeV}$, was carefully analysed with no observed SUSY signal. In an attempt to further increase the sensitivity of the search, the four groups combined some of their searches, to no avail.
\end{abstract}

Keywords. SUSY; LEP; ALEPH; DELPHI; L3; OPAL; minimal supersymmetric standard model; gauge-mediated supersymmetry breaking.

\section{Apology}

It is impossible to cover in one talk the numerous searches that have been carried out by the four LEP Collaborations at LEP-2 for the various possible SUSY signals. Actually, it is even impossible to quote them all. Instead, some of the LEP-combined results will be quoted here and an emphasis will be put on the wide variety of searches.

One does not know how supersymmetry is broken. The details of the soft symmetry breaking determine the way SUSY will exhibit itself and therefore one actually does not know how one should look for SUSY. The fact that so many channels have been studied increases our confidence that none of the SUSY signals has escaped LEP searches.

The interpretation of most of the LEP searches was done in the framework of the MSSM. Some results were discussed in the framework of gauge-mediated symmetry breaking (GMSB) or MSSM with RPV and a few in more exotic variants of SUSY. Consequently, most of the results which are quoted below have been done in the framework of MSSM unless otherwise mentioned. All the results which are stated here are still preliminary.

\section{Search for MSSM signals}

\subsection{The search for sleptons}

The simplest search is the one for sleptons. Here LEP has no competitors at all. The pair of sleptons gives rise to a final state of two acollinear leptons. It is easy 


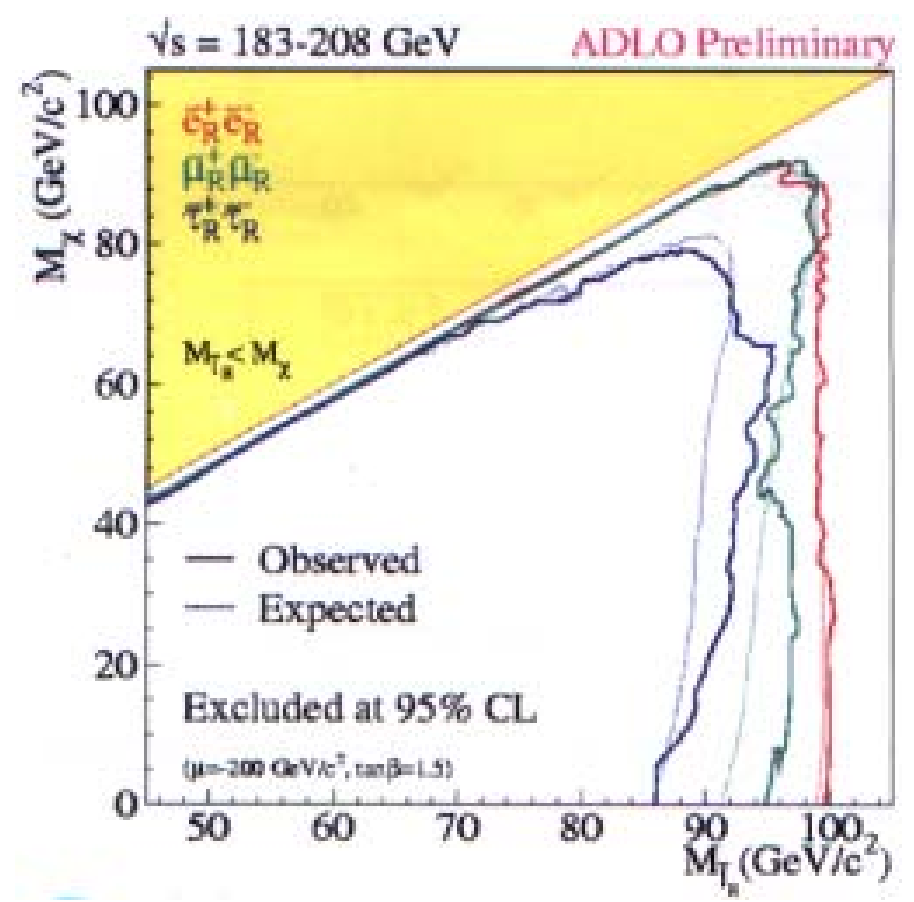

Figure 1. Combined LEP mass limits on sleptons in MSSM based on all the data collected with energy between 183 and $208 \mathrm{GeV}$.

to observe such a final state as long as the mass difference between the slepton and the neutralino (the LSP) is large enough to leave the leptons with enough energy. Once the mass difference is small the final state becomes harder to see, which is the reason for the gap along the diagonal in figure 1 [1].

For $0<m_{\chi_{1}^{0}}<40$ one obtains the mass limits given in table 1 .

A significant effort was invested in reducing the size of the gap along the diagonal. Some searches were limited to a single lepton instead of the two, allowing one to escape detection. In the case of selectrons, a combination of the direct search for selectrons, for charginos and for neutralinos could be combined [2] to obtain an absolute lower mass limit on the $\tilde{e}_{\mathrm{R}}\left(\tilde{e}_{\mathrm{L}}\right)$ of $73(107) \mathrm{GeV}$.

Table 1. Mass limits on MSSM sleptons with relatively light $\chi_{1}^{0}$ and GMSB limits for $N<6$.

\begin{tabular}{lccc}
\hline Slepton & Observed mass limit & Expected limit & GMSB limits \\
\hline$\tilde{e}$ & 99.4 & 99.2 & 65.8 \\
$\tilde{\mu}$ & 94.9 & 91.4 & 96.3 \\
$\tilde{\tau}$ & 85.0 & 84.7 & 86.9 \\
\hline
\end{tabular}



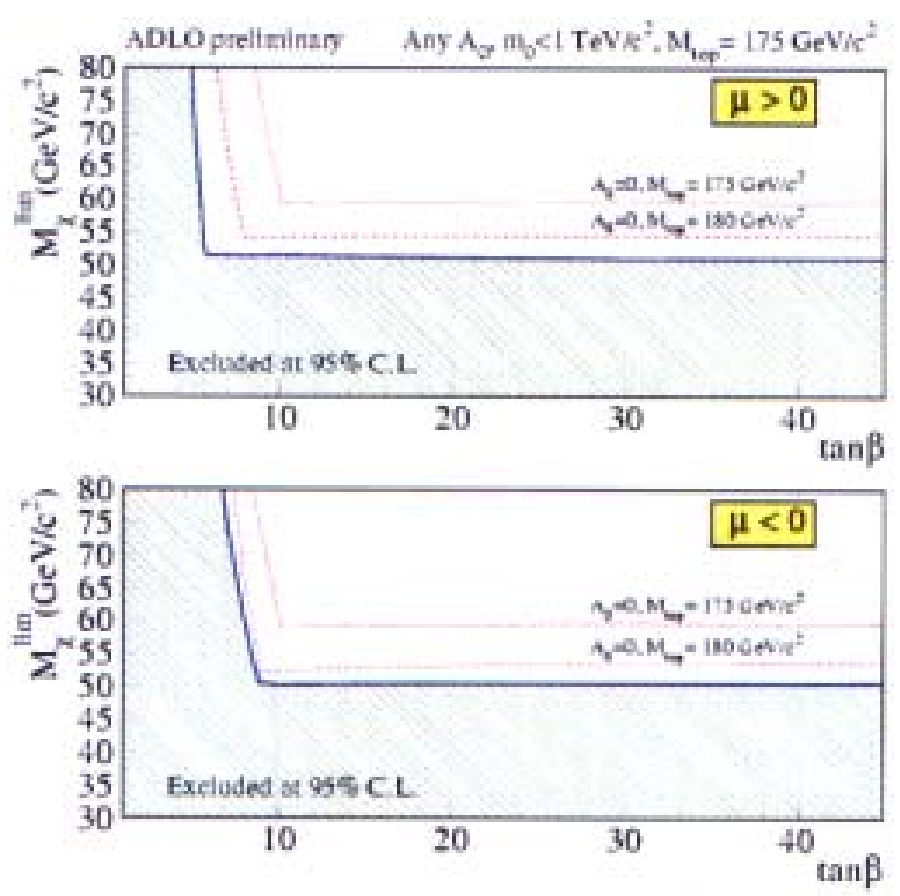

Figure 2. Combined LEP mass limits on the neutralino (LSP) mass in the mSUGRA framework.

\subsection{The search for chargino}

The pair of charginos should give rise to a final state with either a pair of collinear leptons, or a lepton, a pair of jets and a relatively large missing energy, or to a fourjet final state. However, some edges in the parameter space necessitated the study of additional channels (see table 2). When all channels and all LEP experiments are put together, the lower mass limit on the chargino is $m \chi_{1}^{ \pm}>91.9 \mathrm{GeV}[1]$.

\subsection{The search for the neutralino}

The lightest neutralino is also the lightest SUSY particle (LSP) and, hence, of special importance. The combined limit depends on the VEV ratio $\tan \beta$. It is high for low values of $\tan \beta$ and its asymptotic value for high values of $\tan \beta$ and $m_{\text {top }}=175 \mathrm{GeV}$ (the limit drops by $6 \mathrm{GeV}$ for $m_{\text {top }}=180 \mathrm{GeV}$ ), and for $m_{0}<1$ $\mathrm{TeV}$ is set at $50.3 \mathrm{GeV}$ (figure 2) [1].

\section{Search for GMSB signals}

GMSB offers a different phenomenology in which final states are expected to be rich in either photons or tau leptons depending on the identity of the next-to-lightest SUSY particle (NLSP). The masses, and therefore the observable signals depend 


\section{Ehud Duchovni}

Table 2. A very partial list of the final states which were studied by the various SUSY searches.

\begin{tabular}{ll}
\hline Final state & Search channel \\
\hline$\gamma \nu$ & $\chi_{1}^{ \pm}$, GMSB \\
$e \nu$ & $\tilde{l}^{+} \tilde{l}^{-}$ \\
$l^{+} l^{-} \nu$ & $\tilde{l}^{+} \tilde{l}^{-}, \chi_{1}^{ \pm}$ \\
$\gamma \gamma \nu$ & $\mathrm{GMSB}$ \\
$l^{+} l^{-}$(quasi-stable) & $\chi_{1}^{ \pm}$ \\
$l^{+} l^{-} \nu$ & $\tilde{l}^{+} \tilde{l}^{-}$ \\
$l^{+} l^{-} \gamma \nu$ & $\tilde{l}^{+} \tilde{l}^{-}$ \\
$l, 2-$ jets, $\nu$ & $\chi_{1}^{ \pm}$ \\
$l^{+} l^{-} \gamma \gamma \nu$ & $\tilde{l}^{+} \tilde{l}^{-}$ \\
$l^{+} l^{-} l^{+} l^{-} \nu$ & $\tilde{l}^{+} \tilde{l}^{-}$ \\
$l^{+} l^{-}$jet-jet & squarks \\
4 -jets, $\nu$ & $\chi_{1}^{ \pm}$ \\
6-leptons, $\nu$ & $\mathrm{RPV}$ \\
multi-jets + leptons & $\mathrm{RPV}$ \\
\hline
\end{tabular}

mostly on two parameters $\tan \beta$ and $\Lambda$ [3]. The coupling of the NLSP to the LSP is controlled by another parameter of the model and may give rise to a long-lived NLSP. Such a scenario led to various searches for stable and quasi-stable particles. The lower mass limits on the sleptons are given in table 1. LEP also derived lower limits on $\Lambda$ which are assumption-dependent and are typically at a level of a few tens of $\mathrm{TeV}$.

\section{Search for RPV signals}

Quite a few searches for SUSY final states in which an MSSM state is produced and decays through one of the three RPV terms were carried out by LEP. Final states are usually complex with a large number of hadronic jets and leptons. The mass limits are typically at $100 \mathrm{GeV}$ or on the relevant couplings, namely, $\lambda$.

\section{Conclusion}

It looks as if not a single channel has been left untouched and SUSY, if indeed this is the way nature chooses it, is beyond the reach of LEP.

\section{References}

[1] http://lepsusy.web.cern.ch/lepsusy/

[2] ALEPH Coll.: Phys. Lett. B544, 73-88 (2002)

[3] See e.g., S Dimopoulos, S Thomas and J D Wells, Nucl. Phys. B488, 39-91 (1997) 